diastolic blood pressure: $97 \pm 15 \mathrm{mmHg}$ ). The prevalence of LAE by CMR was $26 \%$ and by ECG varied from $1 \%$ (P-mitrale) to $27 \%$ $\left(\mathrm{P}\right.$ axis $<30^{\circ}$ ), and was $46 \%$ when $\geq 1$ ECG LAE criteria were present. There was no significant difference in mean LAVI when $\geq 1$ ECG LAE criterion was present compared to when no ECG LAE criteria were present $\left(47 \pm 15\right.$ vs $\left.50 \pm 15 \mathrm{ml} / \mathrm{m}^{2}, \mathrm{p}=0.235\right)$. All the individual ECG LAE criteria were more specific than sensitive (Table 1/A), with specificities ranging from $70 \%$ ( $\mathrm{P}$ axis $<30^{\circ}$ ) to $99 \%$ (P-mitrale). Obesity attenuated the specificity of most of the individual ECG LAE criteria (Table 1/B). Obesity correlated with significant lower specificity $(48 \%$ vs $65 \%, \mathrm{p}<0.05)$ and a trend towards lower sensitivity ( $59 \%$ vs $43 \%, \mathrm{p}=0.119)$ when $\geq 1$ ECG criteria of LAE were present.

Conclusion Individual ECG criteria of LAE in hypertension are specific, but not sensitive, for identifying anatomical LAE, relative to CMR. LAE in hypertension should not be excluded on the basis of the ECG, particularly in obese subjects.

\section{GLOBAL LONGITUDINAL STRAIN USING FEATURE TRACKING IDENTIFIES THE PRESENCE OF CHRONIC MYOCARDIAL INFARCTION IN PATIENTS WITH NORMAL LV EJECTION FRACTION}

*G Fent, P Garg, L Dobson, T Al Musa, J Foley, J Greenwood, S Plein, P Swoboda. Leeds Institute for Cardiovascular and Metabolic Medicine, University of Leeds, Worsley Building, Clarendon Way, Leeds, LS2 9JT UK

\subsection{6/heartjnl-2016-309668.9}

Objectives Chronic myocardial infarction (MI) detected by late gadolinium enhancement (LGE) is associated with significant mortality and morbidity. Limited subendocardial infarction may not lead to reduction in ejection fraction (EF) and a regional wall motion abnormality (RWMA) may not be evident. Global longitudinal strain (GLS) is impaired independently of EF in a number of conditions, enabling early detection of disease. Strain imaging predicts final infarct size in MI and is superior to LVEF in predicting morbidity and mortality. We hypothesised subjects with chronic MI but normal EF would have impaired GLS compared to healthy volunteers.

Methods Twenty patients with chronic MI (defined as subendocardial hyperenhancement on LGE) and normal LVEF and 20 healthy volunteers underwent CMR at either $1.5 \mathrm{~T}$ or $3.0 \mathrm{~T}$ (Philips Achieva TX). Standard bSSFP cine images were used to calculate LV dimensions and GLS by feature tracking (CVI 42,
Circle Cardiovascular Imaging Calgary, Canada). LGE imaging was performed in all patients $(0.2 \mathrm{mmol} / \mathrm{kg}$ Gadolinium DTPA).

Results Patients were matched for age $(59.8 \pm 12$ vs $59.6 \pm 5.4$ $\mathrm{p}=0.95)$ and $\mathrm{EF}(60.4 \pm 3.8$ vs $62.2 \pm 3.5 \mathrm{p}=0.11)$. Visual evidence of RWMA was present in 13/20 (65\%) of chronic MI patients and $0 / 20$ healthy volunteers. GLS was significantly lower in patients with chronic MI than in those without $(-16.07 \pm 3.9$ vs $-19.79 \pm 2.3 \mathrm{p}=0.001)$ (Figure 1$)$.

Conclusion GLS is impaired in patients with chronic MI but normal LVEF. GLS identifies abnormalities in LV systolic contraction not apparent with EF alone. It may reveal chronic MI in patients with contraindications to gadolinium-based contrast or prognostication of this subset of chronic MI patients. GLS could be used to detect chronic MI by alternative imaging modalities.

\section{QUANTITATIVE MYOCARDIAL PERFUSION AND LONGITUDINAL STRAIN BY FEATURE TRACKING IN NEWLY DIAGNOSED, TREATMENT NAÏVE RHEUMATOID ARTHRITIS}

${ }^{*}$ G Fent, P Garg, L Dobson, T Al Musa, J Foley, P Swoboda, J Greenwood, S Plein. Leeds Institute for Cardiovascular and Metabolic Medicine, University of Leeds, Worsley Building, Clarendon Way, Leeds, LS2 9JT UK

\subsection{6/heartjnl-2016-309668.10}

Objectives Rheumatoid arthritis (RA) is associated with increased cardiovascular mortality. Proposed mechanisms include coronary microvascular dysfunction due to immune dysregulation and systemic inflammation.

First pass myocardial perfusion CMR allows quantification of myocardial blood flow (MBF) and myocardial perfusion reserve (MPR). In the absence of coronary artery disease (CAD), reduced MPR represents coronary microvascular dysfunction. We hypothesised MPR would be reduced in RA and that abnormalities in left ventricular (LV) deformation would be evident in RA, as LV mass has been reported to be reduced in established disease.

Methods Twelve patients with newly diagnosed, treatment naïve RA and 12 healthy volunteers (HV) underwent CMR at 3.0T (Phillips Achieva TX). Both groups had no history of CAD. Dual bolus resting and stress perfusion imaging was performed $(0.1 \mathrm{mmol} / \mathrm{kg}$ Gadolinium DTPA) and MBF estimated for the mid ventricular slice using Fermi constrained convolution (PMI v 0.4 [Sourbron, 2009]). Left ventricular ejection fraction (LVEF) and global longitudinal strain (GLS) by feature tracking

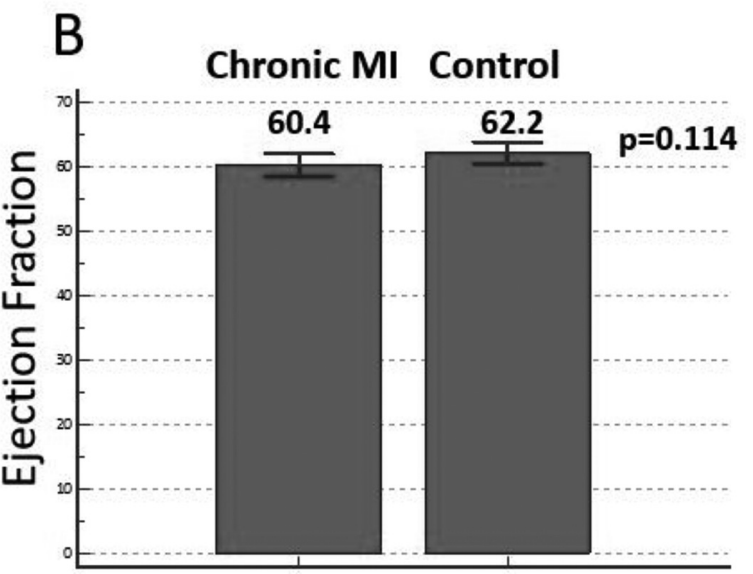

Abstract 9 Figure 1 Graphs showing (A) GLS and (B) LVEF in chronic MI vs controls 

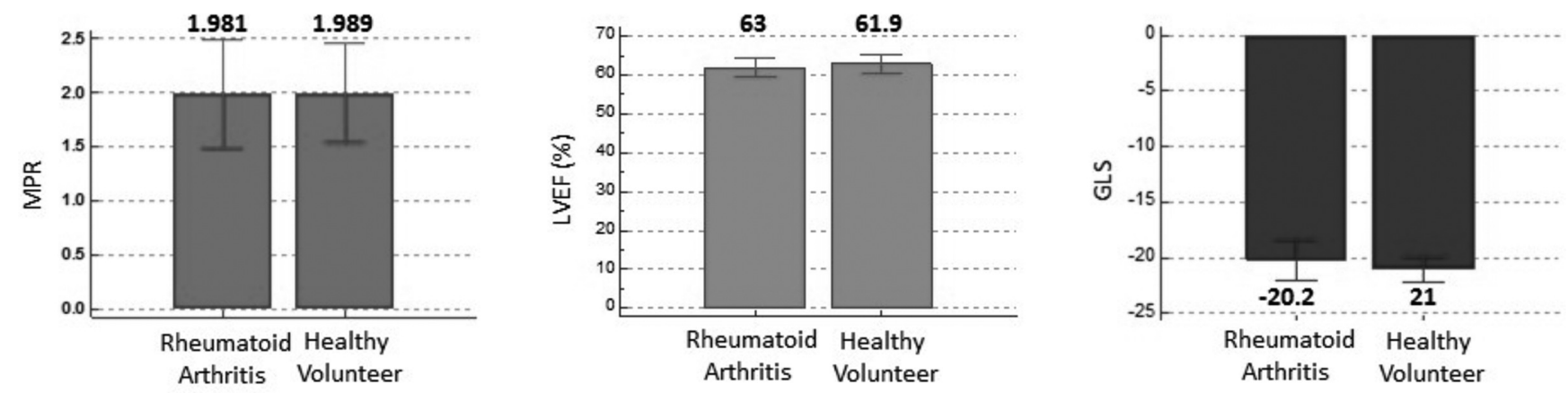

Abstract 10 Figure 1 Graphs illustrating mean values for MPR, LVEF and GLS in RA and HV

were calculated using bSSFP images (CVI ${ }^{42}$, Circle Cardiovascular Imaging, Calgary, Canada).

Results Mean age of RA patients was $48 \pm 16$ and mean age of HV $47 \pm 14$. Of the RA patients, 4 were male and 8 female. Of the HV, 2 were male and 10 female. Mean MPR values were 1.98 \pm 0.79 and $1.99 \pm 0.72(\mathrm{P}=0.88)$ for $\mathrm{RA}$ and $\mathrm{HV}$ respectively (Figure 1). Mean values for LVEF were $63 \pm 4$ and $62 \pm 4 \%$ (P = 0.48 ) respectively for RA patients and HV. Mean values for GLS were -20.2 and $-21 \pm 0.4(\mathrm{P}=0.396)$ for $\mathrm{RA}$ and $\mathrm{HV}$ respectively.

Conclusion These findings suggest treatment naïve RA patients have no detectable abnormalities on perfusion CMR. Therefore, whilst present in established RA, coronary microvascular dysfunction may not yet have developed in early RA. No abnormalities of LV systolic function were evident and may be a later manifestation of RA.

\section{LEFT VENTRICULAR END DIASTOLIC FILLING PRESSURE PREDICTED BY LEFT ATRIAL STRAIN MEASURED BY FEATURE TRACKING}

*JRJ Foley, P Garg, TA Musa, LE Dobson, PP Swoboda, GJ Fent, P Haaf, S Plein, JP Greenwood. LICAMM \& MCRC, University of Leeds, UK

\subsection{6/heartjnl-2016-309668.11}

Introduction Left ventricular end-diastolic filling pressure (LVEDP) is an invasive measure of LV function obtained at cardiac catheterisation (CC) that predicts prognosis and guides therapeutic strategy. Echocardiographic E/E' ratio has been shown to be inaccurate for estimation of LVEDP. Feature-tracking cardiovascular magnetic resonance (FT-CMR) is a novel method for quantification of myocardial deformation and can be used to quantitatively assess left atrial (LA) function. Currently there is no validated MRI parameter that estimates LVEDP. We hypothesised that LA strain correlates to LVEDP.

Methods 14 patients in sinus rhythm, with severe AS underwent a 1.5T CMR protocol (Ingenia, Phillips Healthcare, Best, The Netherlands). LVEDP was recorded at the time of CC by standard techniques. 4 chamber and mid ventricular short axis steady state free procession cine images were obtained: LA endocardial and epicardial borders were traced manually on the end-diastolic slice and strain measurements were calculated using commercially available post-processing software (CVI42, Circle Cardiovascular Imaging, Calgary, Alberta, Canada).

Results Patients were divided into 2 groups: low EDP (13 \pm $2.4 \mathrm{mmHg})$ and high EDP $(36.1 \pm 3.4 \mathrm{mmHg})(\mathrm{p}<0.01)$. Both groups were evenly matched for baseline demographics (Table 1). Peak atrial longitudinal strain (PALS) was significantly different between low EDP and high EDP group $(-21.7 \pm 8.5$ versus $-11.1 \pm 2.1 \% \mathrm{p}=0.01$ ) (Figure 1 ). In multivariable analysis of demographics and CMR parameters PALS was the only determinant of LVEDP independent of other factors (Beta $-0.93 \mathrm{p}=$ 0.01). There was a moderate negative correlation between increasing invasive LVEDP and PALS (Pearson's correlation coefficient $-0.647, \mathrm{p}=0.009$ ).

Conclusion LA function (PALS) as measured by FT-CMR is independently associated with LVEDP and may have a role in predicting LV filling pressures via a routine CMR protocol.

\begin{tabular}{llll} 
Abstract 11 & Table 1 & \multicolumn{3}{l}{ Baseline demographics } \\
\hline & Low EDP $(\mathbf{n}=\mathbf{7})$ & High EDP $(\mathbf{n}=7)$ & p-Value \\
\hline Age (years) & $80.2 \pm 5.6$ & $77 \pm 5.0$ & 0.38 \\
Hypertension (\%) & 71.4 & 57.1 & 0.83 \\
MI (\%) & 42.9 & 57.1 & 0.73 \\
DM (\%) & 14.3 & 14.3 & 0.93 \\
PPG (mmHg) & $92.5 \pm 20$ & $84.4 \pm 9.22$ & 0.45 \\
LVEF (\%) & $58.9 \pm 6.55$ & $57.8 \pm 8.1$ & 0.81 \\
PALS (\%) & $-21.7 \pm 8.5$ & $-11.1 \pm 2.1$ & 0.01 \\
\hline
\end{tabular}

LVEF: left ventricular ejection fraction; MI: previous myocardial infarction; DM: Diabetes mellitus; PPG: peak pressure gradient; PALS: peak atrial longitudinal strain

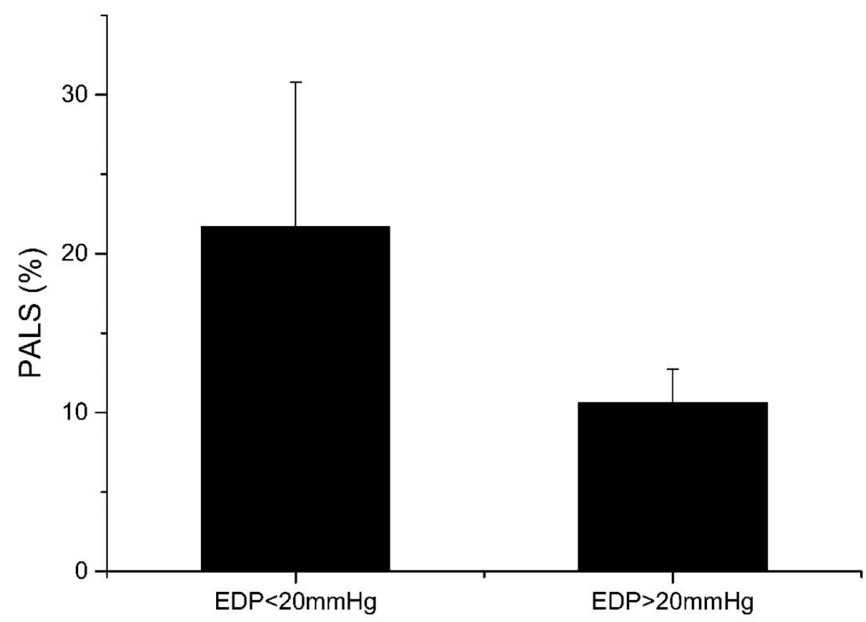

Abstract 11 Figure 1 PALS grouped by invasive LVEDP (mean and SD error bars) 